SESSION 7

MAGNETIC SHEAR AND ELECTRIC CURRENTS 


\title{
MODEL PREDICTIONS FOR MAGNETIC SHEAR CHANGES DURING SOLAR FLARES
}

\section{T.G. FORBES}

Institute for the Study of Earth, Oceans, and Space, University of New Hampshire, Durham, NH 03824, USA

\begin{abstract}
Magnetic shear is sometimes defined observationally as the rotation of the photospheric vector magnetic field away from the field direction of a corresponding potential state. It is often assumed that the obser vational shear is a direct measure of the energy stored in coronal currents. However, this is not true in general as is illustrated here by considering the changes in observational shear in a variety of flare models. Some of these models predict rotations in the photospheric transverse field which are too small to be detected by present day instruments, but other models predict relatively large rotations in highly localized regions. In principle, observations of the transverse field might confirm or eliminate models in the latter group.
\end{abstract}

\section{INTRODUCTION}

Recently, Wang (1992) has reported rapid changes in the polarization of photo spheric Zeeman lines during the onset of flares. Wang interprets these changes as variations in the transverse component of the photospheric magnetic field, although the possibility remains that the changes are due to non-magnetic effects. The inferred field changes are characterized in terms of a 'shear' parameter $S$ which Wang defines as

$$
S \equiv\left|B_{t}\right| \theta
$$

where $\left|B_{t}\right|$ is the magnitude of the transverse magnetic field and $\theta$ is the angle between the observed transverse field and the transverse field computed by potential extrapolation of the observed longitudinal field as illustrated in Figure 1. In this figure the solid curve is the observed transverse field, while the dashed line is the transverse field computed from the longitudinal field (circles with + and signs) by assuming a potential (i.e. current-free) corona. Quotation marks are used in referring to $S$ as the 'shear' parameter because the definition of $S$ as shear 
is not the same as that used by theorists who define shear as occurring whenever $\nabla \times \mathbf{B} \neq \mathbf{0}$.

Since the polarization measurements can only determine the magnitude and orientation of the transverse field, the sign of the field is ambiguous. Consequently, for any set of measurements $\theta$ has two possible values, one greater than $90^{\circ}$ and the other less than $90^{\circ}$.

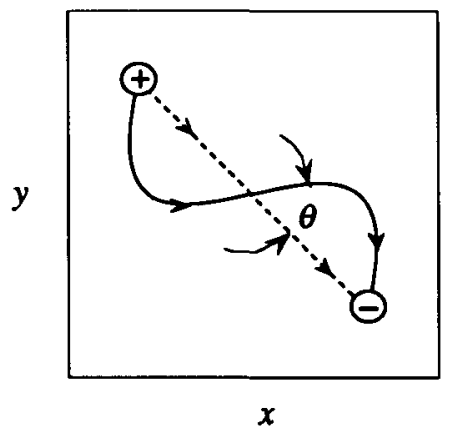

Fig. 1. Definition of the shear angle $\theta$ in the $x-y$ plane of the photosphere.

Wang (1992) further defines a mean weighted 'shear' angle, $\bar{\theta}$ for an entire active region as

$$
\bar{\theta} \equiv \frac{\Sigma\left|B_{t}\right| \theta}{\Sigma\left|B_{t}\right|}
$$

where $\Sigma$ indicates a sum overall points in the active region. The angle $\theta$ at each point is weighted by the corresponding magnitude of $B_{l}$ in order to reduce the noise in the mean angle $\bar{\theta}$. By weighting $\theta$ in this manner, those points where $B_{t}$ is too small to be accurately measured do not contribute significantly to the mean angle.

Wang and Zirin report that $\bar{\theta}$ increases within 5 to 10 minutes after the on set of flares which is puzzling because most models predict that $\bar{\theta}$ should decrease during this period. As is discussed in the subsequent sections, most flare models predict that $\bar{\theta}$ should increase prior to the flare as the field in the corona becomes less and less potential due to the gradual build-up of current in the corona. When the flare starts, this current is dissipated, and the field becomes more potential. Consequently, $\bar{\theta}$ should decrease.

\section{MAGNETIC ENERGY OF A FORCE-FREE FIELD}

As has been pointed out by Low (1985), the free magnetic energy, $W$, of a forcefree field in the corona can be expressed solely in terms of the vector magnetic field at the surface of the Sun. If the surface lies in the $x-y$ plane, with $z$ being the radial outward direction, then $W$ is given by

$$
W \equiv \frac{1}{8 \pi} \iiint_{z>0} \Delta B^{2} \mathrm{~d} x \mathrm{~d} y \mathrm{~d} z=\frac{1}{4 \pi} \iint_{z=0}\left(x \Delta B_{x}+y \Delta B_{y}\right) B_{z} \mathrm{~d} x \mathrm{~d} y
$$

where $\Delta \mathbf{B}=\mathbf{B}-\mathbf{B}_{\mathbf{p}}$ is the difference between the total magnetic field $\mathbf{B}$ and the potential magnetic field $\mathbf{B}_{\mathbf{p}}$, and $z=0$ is the surface of the Sun. The field $\mathbf{B}_{\mathbf{p}}$ is the component of the coronal field due to current sources below the surface, and the field $\Delta B$ is the field due to the coronal currents alone. Thus, the free energy $W$, which is the energy available for a flare, can be expressed directly in terms of the 
field components at the surface $z=0$. In general, equation (3) is not valid if current sheets are present in the corona since such sheets are not force-free. Equation (3) is also not valid for an infinitely long, two-dimensional configuration because the expression assumes that the fields and currents at infinity are zero.

Assuming, for convenience, that the potential magnetic field lies in the $x$ direction (i.e. $B_{p y}=0$ ), then the free energy can be expressed in terms of the angle $\theta$ and the transverse magnetic $B_{t}$ as

$$
W=\frac{1}{4 \pi} \iint_{z=0}\left[x\left(B_{t} \cos \theta-B_{t p}\right)+y B_{t} \sin \theta\right] B_{z} d x d y
$$

where $B_{t p}$ is the transverse potential field. Equation (4) shows that the magnetic energy stored in the corona depends on both the shear angle $\theta$ and the transverse magnitude, $B_{t}$. The energy stored in the corona and released during a flare depends upon more than just the 'shear' angle $\theta$. Even if all changes in the trans verse field were due to rotations, it is the rotations occurring where $B_{t} B_{z}$ is large that are important, rather than the rotations occurring where $B_{t}$ alone is large. Thus, the use of the weighting factor $B_{t}$ in (2) also weakens the connection between the weighted 'shear' angle $\bar{\theta}$ and the magnetic energy stored in the corona.

\section{FLARE MODELS}

Changes in the transverse field at the photosphere should occur during all flare phases unless surface currents exist which shield the photosphere from the currents in the corona. In the absence of such shielding currents, vector magnetograph measurements can, in principle, distinguish between various flare models.

Most flare models are based on mechanisms which release magnetic energy stored in the corona. The energy is stored in the form of coronal currents, and energy is released when these currents become unstable due to the slow evolution of the normal magnetic field component at the photosphere. Large eruptive flares typically release about $10^{32}$ ergs, most of which is associated with the kinetic energy of the ejecta. Releasing this amount of energy requires only modest changes in the coronal magnetic field. For example, a decrease in the coronal magnetic field from 100 Gauss to 90 Gauss in a volume with a scale-length of $10^{5}$ $\mathrm{km}$ (i.e the scale size of ejecta) releases $8 \times 10^{31} \mathrm{ergs}$. If the change in the field is spread uniformly throughout the volume, then the average 'shear' angle $\theta$ is of order $\tan ^{-1}(10$ Gauss $/ 100$ Gauss $)=5.7^{\circ}$. This value is relatively small and cannot easily be observed with current vector magnetographs (Klimchuk et al. 1992). However, the changes in $\theta$ are not necessarily uniform, and some flare models predict localized region where $\theta$ varies by as much as $180^{\circ}$.

\section{Dynamo Model}

Unlike most flare models, the dynamo model proposed by Sen and White (1972) and Kan et al. (1983) requires an increase in the magnetic energy of the 
corona during the flare. In this model the actual energy source of the flare is a dynamo region hidden in the convection zone below the photosphere, and a flare occurs when a field aligned current is produced in a loop by the sudden motion of the plasma in the dynamo region. The increase in the loop current drives the loop outwards, and the dissipation of the current in the loops heat the corona and the chromosphere. This dynamo model does not address the cause of the sudden motions of the plasma in the convection zone, and thus it does not really explain the flare mechanism. Instead, it simply transfers the flare mechanism from the corona to the convection zone.

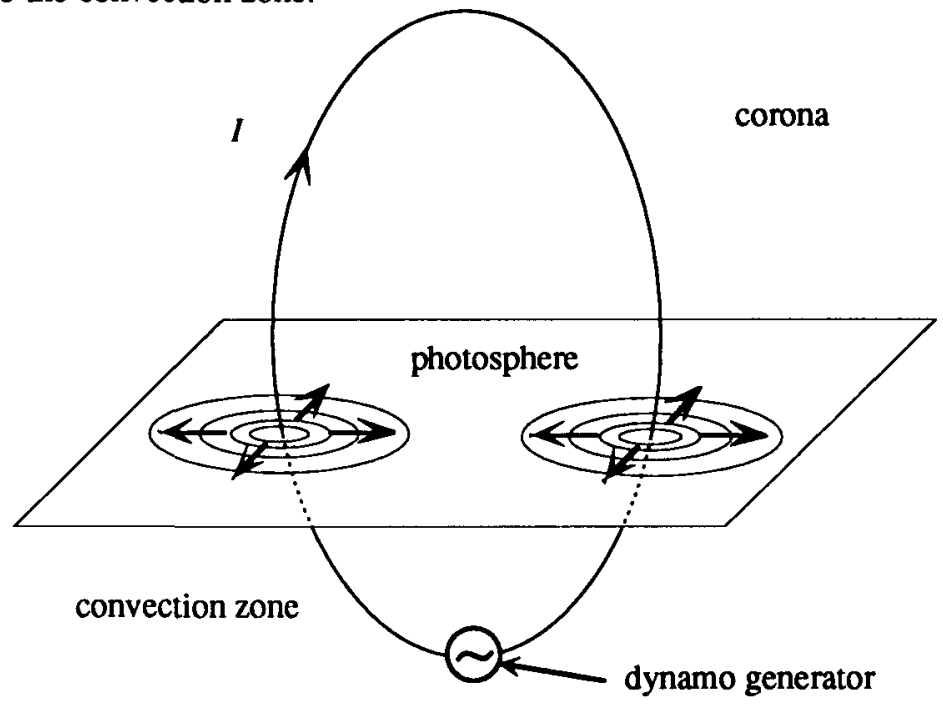

Fig. 2. Photospheric motions produced by the dynamo model during the impulsive phase of the flare. Such motions have never been observed.

The dynamo model predicts that the shear in the corona increases during a flare, and thus it provides a simple explanation for the increases in the 'shear' parameter observed by Wang (1992). However, the dynamo model also predicts that large scale horizontal motions should occur at the photosphere during the impulsive phase of the flare. Although the photosphere is only weakly ionized, it is still an excellent conductor, and field lines there are frozen to the plasma. Thus any sudden enhancement of current flowing from the convection zone to the corona must necessarily move the photospheric plasma as shown in Figure 2. Power transmission from the dynamo region to the corona cannot occur if such motions are suppressed. Analyses by Melrose and McClymont (1987) and McClymont and Fisher (1989) show that dynamo models require photospheric velocities of almost a $100 \mathrm{~km} / \mathrm{s}$ in order to supply the energy flux for a large flare. Such velocities are two orders of magnitude higher than observed.

Most models based on the storage of magnetic energy prior to the flare have a dynamo process, but this process occurs during the pre-flare growth phase 
rather than the impulsive phase. Many storage models assume that the corona is initially current free and that the build-up of magnetic energy is entirely due to stressing of the coronal field by the observed photospheric motions. These motions are on the order of $1 \mathrm{~km} / \mathrm{s}$ or less, and over a time period of several days they are sufficient to store the $10^{32}$ ergs needed for a large flare. However, large flares have sometimes been observed in regions where the photospheric motions are too slow or of too short a duration to store $10^{32}$ ergs. These flares imply that the magnetic fields emerging from the convection zone may not be current free and may already be in a stressed state (McClymont and Fisher 1989).

\section{Arcade Models}

Observations by Hagyard (1988) and others show that large flares often occur in arcades of strongly sheared loops, and considerable theoretical work is in progress to understand how such sheared arcades might erupt. Figure 3 shows an example of a two-dimensional, infinitely long arcade of width $2 L$ with $|x|<L$ having the field components:

$$
\begin{gathered}
B_{x}=\left(l B_{o} / k\right) \cos k x e^{-l z} \\
B_{y}=\left(\alpha B_{o} / k\right) \cos k x e^{-l z} \\
B_{z}=-B_{o} \sin k x e^{-l z}
\end{gathered}
$$

where $l^{2}=k^{2}-\alpha^{2}$ and $k=\pi /(2 L)$. The footpoints of the arcade are displaced a distance $d(x, t)$ in time $t$ at a velocity $V_{y}=V_{o} x / L$ along a line parallel to the $x$ axis. The magnetic energy, $W_{m}$, per unit length increases as

$$
W_{m}=B_{o}{ }^{2} L^{2}(2 \pi)^{-2}\left(1+V_{o}{ }^{2} t^{2} L^{-2}\right)^{1 / 2}
$$

where $\mu$ is the magnetic permeability of free space.

No loss of ideal-MHD equilibrium or stability occurs in the above arcade as

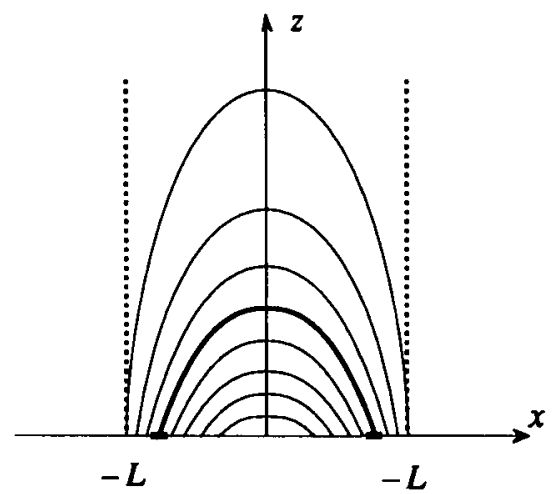

(a)

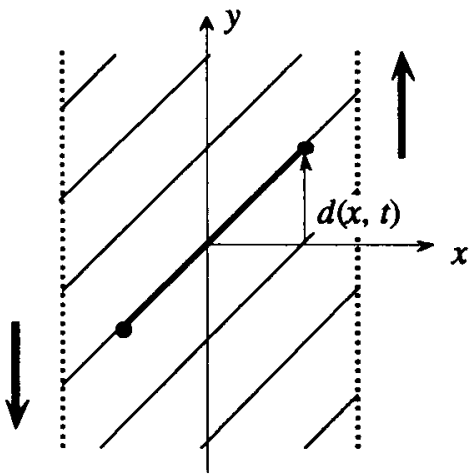

(b)

Fig. 3. Field lines for a periodic, linear force-free arcade in vertical (a) and horizontal (b) projections [from Priest and Forbes 1990]. 
the footpoints are displaced, and it is now thought that a simply connected, infinitely long, two-dimensional arcade always remains in a stable equilibrium as the footpoints are sheared. (Finn and Chen 1990a). Whether loss of equilibrium or stability can occur in three-dimensional arcade configurations is not yet established. There does seem to be a growing consensus that if such a loss does exist, it cannot cause the field to become completely open (Aly 1991, 1992, Linker and Mikić 1992).

Inhester et al. (1992) found that infinitely long, two-dimensional arcades can erupt if reconnection occurs. As the arcade is sheared, a vertical current layer develops, and reconnection of field lines in this layer leads to the formation of a magnetic flux rope as shown in Figure 4.

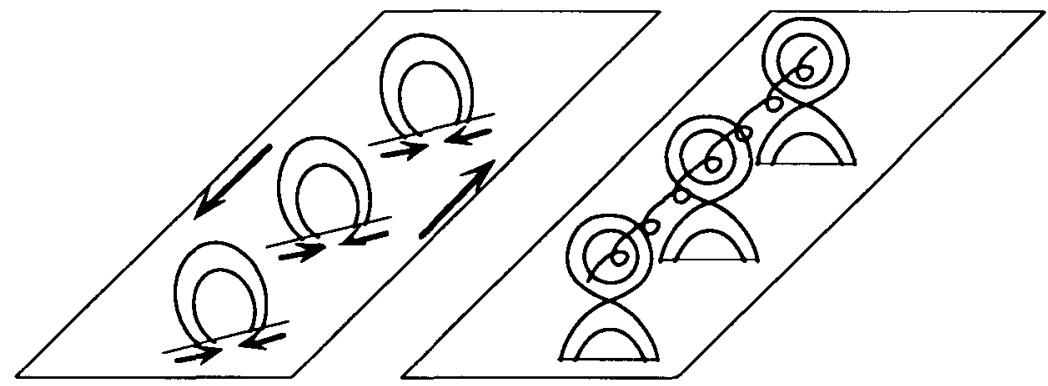

(a)

(b)

Fig. 4. Formation of a flux rope by shearing and reconnection in an arcade [after van Ballegooijen and Martens 1989].

As the flux rope forms, the photospheric field in Figure 4 rotates back to the orientation of the potential field implying that the 'shear' is decreasing even though the magnetic energy stored in the system is increasing. Yet we know from Equation (3) that the non-potential transverse components on the surface must increase ingoing from (a) to (b) even though the orientation of the field at the surface appears to become more potential. The apparent contradiction with Equation (3) is due to the fact that Figure 4 does not show the photospheric regions where the field lines of the flux rope are attached to the surface. The 'shear' in the attachment regions increases as the twisted flux rope is created, so that the field components at the surface increase in accordance with Equation (3).

Once a flux rope is formed, the field is no longer simply connected and idealMHD equilibrium can be lost even in a two-dimensional, infinitely long system. In the model of Forbes and Isenberg (1991), an eruptive flare occurs when the balance between magnetic compression and magnetic tension is lost as shown in Figure 5. Prior to the eruption, there is a balance between the upward force caused by field lines compressed between the flux rope and the photosphere and the downward force caused by the tension in the field lines passing over the top of the flux rope. If the current in the flux rope is increased by twisting the ends of the flux rope, then the magnetic compression force increases, and the flux rope rises. 
Alternatively, if the photospheric flux is reduced by slowly converging and reconnecting the field lines in the photosphere as shown in Figure 5, then the flux rope also rises.

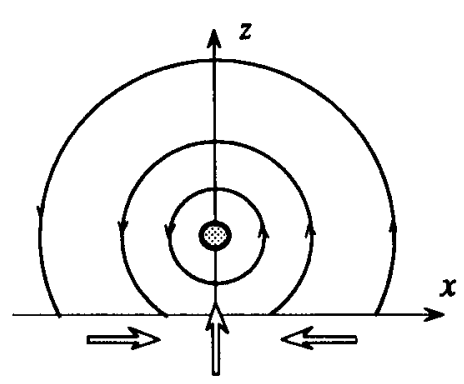

(a)

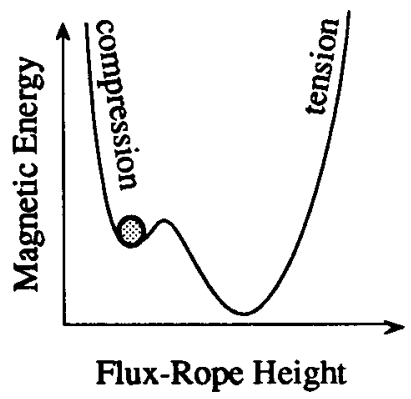

(b)

Fig. 5. Driving mechanism for a model of an eruptive flare based on an arcade containing a flux rope. Slow reconnection of field lines in the photosphere (a) leads to a build of current in the flux rope. As the current increases the magnetic energy hill which separates lower and upper equilibria disappears.

For some boundary conditions, a sudden loss of equilibrium will occur as the photospheric field decreases relative to the flux-rope field. An example is shown in Figure 6 for a quadrupolar photospheric magnetic field with the normal component:

$$
B_{z}(x, y, 0)=Q(t) x\left(3 d^{2}-x^{2}\right)\left(x^{2}+d^{2}\right)^{-3}
$$

where $Q(t)$ is the field strength, $d$ is the scale-length, and $c$ is the speed of light. As $Q(t)$ decreases, the photospheric flux decreases, and the flux-rope height, $h$, slowly increases up to a critical point as shown in Figure 6. At the critical point the flux rope suddenly jumps to a higher equilibrium forming an extended current sheet in the process as shown in Figure 7. Reconnection of this current sheet allows the flux rope to escape.

Numerical simulations suggest that the reconnection in the current sheet below the flux rope occurs very rapidly because the reconnection is driven by the flows generated by the jump (Forbes 1990, 1991). The moving flux rope creates vortical flows which pinch the current sheet until the current sheet becomes thin enough to undergo rapid reconnection. Because the flow towards the current sheet is driven, the reconnection rate depends only weakly on the electrical conductivity of the plasma (Forbes and Priest 1987).

In the two-dimensional flux rope model the 'shear' angle $\theta$, as defined by Wang (1992), remains zero everywhere in the $x-y$ plane. However, the field below the filament actually flips from anti-parallel to parallel relative to the potential field (see Figures 5 and 6). It is only because Wang defines $\theta$ to be always less than $90^{\circ}$ that $\theta$ appears to remain zero. If this restriction were removed, then 


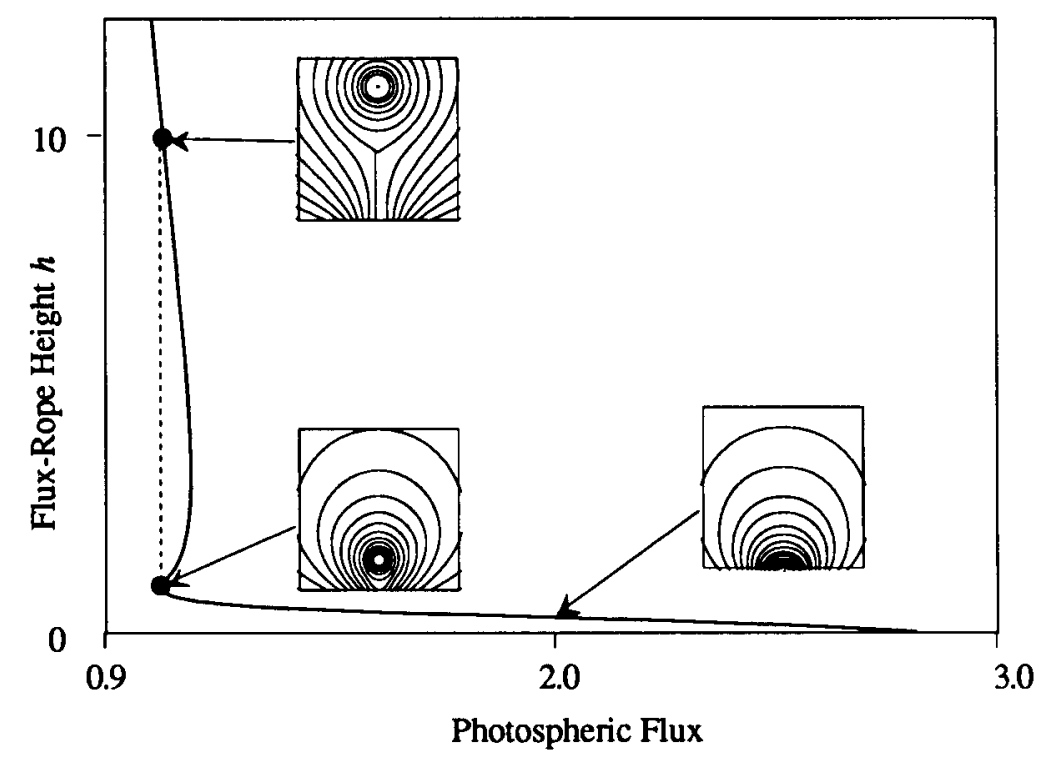

Fig. 6 Equilibrium curve for a two-dimensional flux rope suspended above a quadrupolar photospheric field.

$\theta$ would flip by $180^{\circ}$ during the jump shown in Figure 6. As discussed previously, the angle $\theta$ also changes at the boundaries at $y= \pm \infty$ where the flux rope is attached to the surface.

\section{CONCLUSIONS}

Rotations in the transverse vector magnetic field at the photosphere are insufficient by themselves to determine whether the magnetic energy stored in the corona is increasing or decreasing. If the coronal field is force-free, then it is possible, in principle, to determine the magnetic energy stored in the corona by observing changes in both the orientation and magnitude of the transverse vector magnetic field and combining these with observations of the normal component of the vector field. If current sheets are present in the corona, then measurements of the vector magnetic field at the photosphere are insufficient to determine the magnetic energy stored in the corona.

Even if the currents in the corona are force-free, present day observations are too noisy to determine changes in the magnetic energy of the corona that are expected to occur during flares (Klimchuk 1992). However, this does not neces sarily mean that no changes in the transverse photospheric field can be detected. Although the average change in the coronal magnetic field during a flare is less than $10 \%$, the change in the photospheric field at certain locations could be as much as $100 \%$ according to some flare models. 


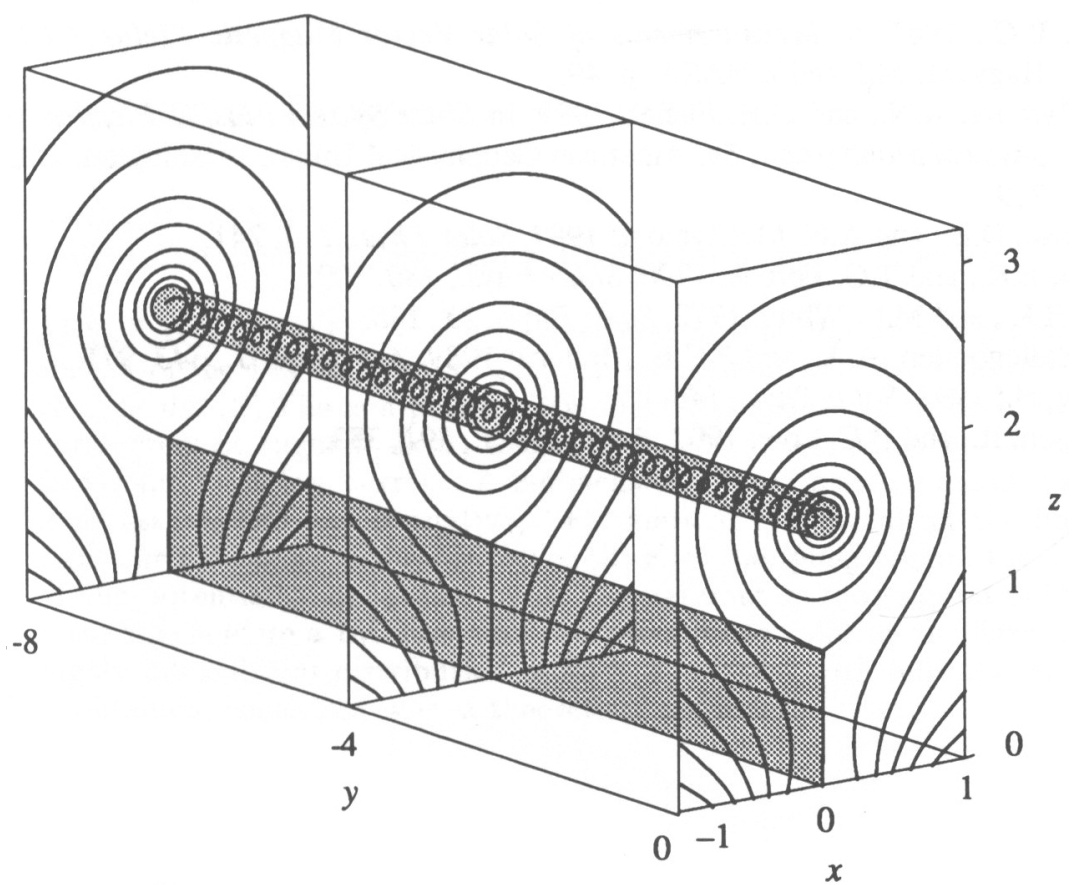

Figure 7- Three dimensional view of the upper equilibrium magnetic field configuration at the critical point.

\section{ACKNOWLEDGEMENTS}

The author is grateful to Hal Zirin, Haimin Wang, and the local organizing committee in Beijing for their financial support and hospitality during the meeting. This work was partially supported by the NASA Space Physics Theory Program through NASA Grant NAG 5-1479 to the University of New Hampshire.

\section{REFERENCES}

Aly, J.J.: 1991, Astrophys. J., 375, L61.

Finn, J.M., and J. Chen: 1990a, Astrophys. J., 349, 345.

Forbes, T.G.: 1990, J. Geophys. Res., 95, 11919.

Forbes, T.G.: 1991, Geophys. Astrophys. Fluid Dynamics, 62, 15.

Forbes, T.G., and P.A. Isenberg: 1991, Astrophys. J., 373, 294.

Forbes, T.G., and E.R. Priest: 1987, Reviews of Geophys., 25, 1583.

Hagyard, M.J.: 1988, Solar Phys., 115, 107.

Inhester, B., J. Bim, and M. Hesse: 1992, Solar Phys., 138, 257.

Kan, J.R., S.-I. Akasofu, and L.C. Lee: 1983, Solar Phys., 84, 153.

Klimchuk, J.A., R.C. Canfield, and J.E. Rhoads: 1992, Astrophys. J., 385, 327.

Linker, J.A., and Z. Mikic': 1992, Bull. of the Amer. Astron. Soc., 24, 733. 
Low, B.C.: 1985, in Measurements of Solar Vector Magnetic Fields, CP2374, Hagyard, M.J. (ed.), NASA, p. 49.

McClymont, A.N., and G.H. Fisher: 1989, in Solar System Plasma Physics, Geophysical Monograph, 54, American Geophysical Union, Washington, D.C., p. 219.

Melrose, D.B., and A.N. McClymont: 1987, Solar Phys., 113, 241.

Priest, E.R., and T.G. Forbes: 1990, Solar Phys., 130, 399.

Sen, H.K., and M.L. White: 1972, Solar Phys., 23, 146.

van Ballegooijen, A.A., and P.C.H. Martens: 1989, Astrophys. J., 343, 971.

Wang, H.: 1992, Solar Phys., 140, 85.

Wolfson, R., and B.C. Low: 1992, Astrophys. J., 391 , 353. 